\title{
Different nutritional approaches in the treatment of irritable bowel syndrome
}

\author{
(1) E. Nüket Ünsal' ${ }^{1}$, (1) Gamze Akbulut ${ }^{2}$, (1) Harun Erdal ${ }^{3}$ \\ 1 University of Health Sciences Turkey, Gülhane Training and Research Hospital, Clinic of Nutrition and Dietetics, Ankara, Turkey \\ ${ }^{2}$ Gazi University Faculty of Medicine, Department of Nutrition and Dietetics, Ankara, Turkey \\ 3University of Health Sciences Turkey, Gülhane Faculty of Medicine, Department of Gastroenterology, Ankara, Turkey
}

\section{Date submitted:}

13.04.2020

Date accepted:

20.05.2020

Online publication date:

15.09.2020

\section{Corresponding Author:}

E. Nüket Ünsal, MD, University of Health Sciences Turkey, Gülhane

Training and Research Hospital, Clinic of Nutrition and Dietetics,

Ankara, Turkey

nuketbuyukgenc@hotmail.com

ORCID:

orcid.org/0000-0002-1491-7093

Keywords: Irritable bowel syndrome, nutrition, fiber, probiotic, FODMAP

\begin{abstract}
Irritable bowel syndrome (IBS) is defined as a combination of pain associated with abdominal discomfort and changes in bowel habits. IBS has an important economic effect on healthcare system and it decreases the life quality of patients. Nutrition treatment is really important to reduce IBS symptoms. In this review, nutritional recommendations are based on scientific literature. The topics which are discussed in this review are low Fermented Oligosaccharide, Disaccharide, Monosaccharide and Polyol Diet, increasing dietary soluble fiber, and probiotic use. Different nutritional approaches are used in the treatment of IBS patients but there is still no clear evidence about them. Therefore, further well-designed, randomized controlled trials are needed.
\end{abstract}

\section{Introduction}

Irritable bowel syndrome (IBS) is defined as a combination of pain associated with abdominal discomfort and changes in bowel habits. IBS is one of the most common functional gastrointestinal diseases that affect about $14 \%$ of the global population (1). Global IBS prevalence varies between $10 \%$ and $20 \%$ depending on the diagnostic criteria and the geographic region (2). Although the etiology is clearly known, changeable bowel motility, visceral hypersensitivity, abnormal brain bowel interaction, nutrient intolerance and altered microflora have been shown to play a role in the IBS (3).

It is known that IBS has negative effects on patients' quality of life. This situation highlights the dietary interventions for treatment (4). Healthy eating and lifestyle suggestions are very important in IBS. Providing hydration and regular physical activity are among the suggestions (5). Besides the regular meal consumption, alcohol, caffeine and spicy food intake should be limited (6). Other nutritional interventions include increasing dietary fiber, regular probiotic use, and a low Fermented Oligosaccharide, Disaccharide, Monosaccharide and Polyol Diet (FODMAP) diet approach (7).

Increasing dietary fiber intake is recommended to alleviate the symptoms of patients with IBS (8). There is no evidence of the optimal fiber dose to be used in IBS, but the target (20-30 $\mathrm{g} /$ day) should be reached in the total dietary fiber (5). Dietary recommendations for patients with IBS include soluble fiber such as psyllium rather than insoluble fiber (9). In the study, it 
has been shown that the consumption of insoluble fiber does not improve IBS symptoms while consumption of soluble fiber improves general IBS symptoms (8).

Probiotics play a major role in the IBS management (4). Probiotics alter colonic fermentation, stabilize the colon microbiota and keep it in balance. They also increase mucosal integrity and improve the intestinal barrier $(10,11)$. As a result of systemic review and metaanalysis in which the effects of probiotics are examined in patients with IBS, it is seen that the use of probiotics results in a reduction in general IBS symptoms (12). However, it is not yet clear which strain and dose are useful in IBS (4).

There are clinical studies supporting that a low FODMAP diet is beneficial for patients with IBS. In these studies, it is observed that there is a reduction in the symptoms such as abdominal pain, gas, bloating and consequently, an increase in their quality of life $(13,14)$.

This article aims to address nutritional approaches that play an important role in the correction of IBS symptoms.

\section{Irritable Bowel Syndrome and Medical Nutrition Therapy}

IBS can be affected by various cultural, social, environmental and behavioral factors as with many functional disorders. Nutrition style, physical activity level, hormonal and psychological effects may increase IBS symptoms. Nutrition is one of the most important factors leading to the exacerbation of symptoms in IBS patients (15). Approximately two out of three patients with IBS think that the symptoms of the gastrointestinal tract are food-dependent (2). $60-70 \%$ of patients with IBS state that the symptoms worsen after meals, $50-70 \%$ of them are unable to tolerate some foods, and more than $70 \%$ of them state that the foods cause some symptoms. For this reason, it is recommended to take a careful nutritional history and conduct dietary interventions when it is necessary in order to determine possible causes of symptoms (16). At the same time, despite its common use, there are limited data supporting the use of special diets in the treatment of IBS symptoms (17).

Standard recommendations for IBS patients include regular meal consumption, regular physical activity, good hydration, and reduction of insoluble fiber, alcohol, caffeine, spicy foods and fat intake (5).

Cozma-Petruţ et al. (5) created a specific nutritional pyramid for IBS. While physical activity and hydration form the basis of the pyramid, the second level emphasizes the correct nutritional habits that can improve health status in IBS and states which food group to consume in what amount per day. The top of the pyramid contains fried and processed foods that should be removed from the diet of IBS patients. Many studies suggest that IBS patients have much more irregular eating habits than the control group, which can affect colonic motility and contribute to IBS symptoms (18).
Some studies emphasize that the focus should be on the elimination diet in order to determine the role of nutrient intolerance in IBS patients. In a study, it was found that approximately half of the women with IBS had symptomatic improvement after a strict 3-week elimination diet that excluded dairy products, cereals, citrus fruits, potatoes, tea, coffee, alcohol, additives and preservatives (2).

\section{a) Fiber}

Dietary fiber is the complex lignin polymers found in undigested carbohydrates and plants that have physiological effects in humans (8). Dietary fiber is divided into two groups as soluble fiber (water-soluble) and insoluble fiber (waterinsoluble). As soluble fiber, pectin in foods such as apple, quince, etc., gums in resin, $\beta$-glucan in foods such as oats etc., and the mucilages are found in plants. From insoluble fiber group, cellulose in wheat, hemicellulose in grains and lignin is abundant in wheat (19).

Dietary fiber has been used in the treatment of various gastrointestinal diseases for many years. Its deficiency can reduce the diversity in the intestinal microbiota, causing disruption of microbiota balance and increase in chronic diseases (20). IBS is thought to be caused by insufficient dietary fiber intake, and it is recommended to increase dietary fiber intake to alleviate the symptoms of patients (8).

Fiber has been considered as the basis of treatment for IBS symptoms for many years and is considered a bulking agent to improve bowel transit and reduce constipation (9). The beneficial effect of fiber is the production of short-chain fatty acid which provides the colon fermentation in order to indicate a prebiotic effect (4). Fermentation of acetate, propionate and butyrate, short-chain fatty acids, which are by-products of dietary fiber, provide the growth of beneficial bacteria such as lactobacilli and bifidobacteria. The effect of fiber is variable in IBS, not all types of pulp are useful (8). Dietary recommendations usually include soluble fiber supplements (9). Supplements which are long chain, medium viscosity, soluble, and moderately fermentable dietary fiber such as psyllium improve the general symptoms of IBS (21).

It is recommended to increase dietary fiber intake to $20-35 \mathrm{~g}$ daily in order to regulate stool and reduce abdominal pain, gas and bloating (8).

The role of fiber in IBS continues to be subject to controversy with high quality evidence and conflicting data. In a systemic review, 12 randomized controlled trials involving 621 patients were evaluated, and the beneficial effect of the soluble and insoluble fiber cannot be found in improving abdominal pain or symptom scores in patients with IBS (5). Twelve systematic meta-analysis showed that patients with IBS who increased dietary fiber intake did not improve IBS symptoms compared to a placebo or low-fiber diet (8). In contrast, in the study 
which examined 14 RCTs that 906 patients participated, it was concluded that the use of psyllium soluble fiber could have beneficial effects on IBS, but the positive effects of insoluble fiber such as bran were not detected or even it could worsen the symptoms (22).

Nowadays, the fiber intake of many people is below the recommended levels. Therefore, changes to dietary habits in order to increase dietary fiber are important for maintaining good health (23).

\section{b) Low FODMAP}

The low FODMAP diet has been developed by Monash University in Australia and is used to treat IBS symptoms based on evidence from prospective studies showing an approximately $75 \%$ improvement in symptoms (1). Increasing evidence supports the effectiveness of the low FODMAP diet in the treatment of IBS (24). The low FODMAP diet reduces gastrointestinal symptoms, improves the quality of life of patients, and positively affects the intestinal microbiota (25).

FODMAPs are fermentable oligosaccharides (wheat, rye, onion and legumes and chicory with garlic and galactans in kernels) and disaccharides (lactose in milk and dairy products), monosaccharides (fructose in artificial sweeteners) and polyols (sugar) (15). FODMAPs are short-chain carbohydrates (1).
Foods are classified into two groups as those with high and low FODMAP contents and they are given in Table 1 (26).

Low FODMAP diet should never be followed in the long term. A 3 or 4-week restriction phase is usually sufficient to get clinical response. If any symptoms improve within 4 weeks of following a low FODMAP diet, intervention should be stopped and other treatment options should be emphasized (5).

Dietary FODMAPs induce hydrogen and methane production in the intestines of IBS patients. Possible changes in intestinal $\mathrm{pH}$ and flora can cause local inflammation. These changes can significantly increase symptoms such as excessive gas and bloating in patients with IBS (1).

In a randomized, controlled, single-blind study by Halmos et al. $(27,28), 30$ patients with IBS and 9 healthy individuals were given 21 days of FODMAP diet or typical Australian diet; $70 \%$ of patients with IBS (all four subtypes of IBS) reported they felt better on the low FODMAP diet. In another randomized controlled study comparing the low FODMAP diet with the high FODMAP diet, it was found that a low FODMAP diet reduced the severity and frequency of abdominal pain in individuals with IBS (29).

If the symptoms improve within 4-6 weeks after applying the low FODMAP diet, the process of adding food back into

\begin{tabular}{|c|c|c|}
\hline & High FODMAP Food Source & Low FODMAP Food Source \\
\hline Excess fructose & $\begin{array}{l}\text { Fruits (mango, apples, pears, nashi pears, sugar snap } \\
\text { peas, clingstone peaches, tinned fruit in natural juice, } \\
\text { watermelon) } \\
\text { Honey } \\
\text { Sweeteners (fructose) }\end{array}$ & $\begin{array}{l}\text { Fruit (durian, banana, blueberry, grape, } \\
\text { carambola, grapefruit, lime, honeydew melon, } \\
\text { kiwifruit, tangelo lemon, mandarin, paw paw, } \\
\text { orange, strawberry passionfruit, raspberry, } \\
\text { rockmelon) } \\
\text { Honey substitutes (maple syrup, golden syrup) } \\
\text { Sweeteners (any except polyols) }\end{array}$ \\
\hline $\begin{array}{l}\text { Lactose } \\
\text { oligosaccharides }\end{array}$ & $\begin{array}{l}\text { Milk (sheep, goat and cow) } \\
\text { Ice cream } \\
\text { Yoghurt } \\
\text { Soft cheeses }\end{array}$ & $\begin{array}{l}\text { Milk (rice milk, lactose-free) } \\
\text { Cheese (camembert, hard cheeses) } \\
\text { Yoghurt (lactose-free) } \\
\text { Ice cream substitutes (sorbet, gelati) } \\
\text { Butter }\end{array}$ \\
\hline $\begin{array}{l}\text { Fructans and/or } \\
\text { galactans }\end{array}$ & $\begin{array}{l}\text { Fruits (apples, longon, apricots, cherries, lychee, nashi } \\
\text { pears, pears, nectarine, peaches, watermelon, plums, } \\
\text { prunes) } \\
\text { Vegetables (avocado, snow peas cauliflower, } \\
\text { mushrooms) } \\
\text { Sweeteners (sorbitol, maltitol, mannitol, xylitol, isomalt) }\end{array}$ & $\begin{array}{l}\text { Fruits (durian, banana, blueberry, raspberry, } \\
\text { carambola, grapefruit, grape, honeydew melon, } \\
\text { lime, mandarin, orange, rockmelon, kiwifruit, } \\
\text { lemon, passionfruit, paw paw) } \\
\text { Sweeteners (sucrose, glucose) }\end{array}$ \\
\hline
\end{tabular}


the diet should be started. The goal at this stage is to be able to create an individually modified FODMAP layout. One of the most important reasons for the inclusion of foods in the diet is that the restricted foods are also prebiotic. Studies have shown that after 3-4 weeks of restricted FODMAP diet administration, there is a negative change in microbiota because of reduced prebiotic intake (29). More detailed studies are needed regarding insufficiencies that a low FODMAP diet can cause (30).

\section{c) Probiotic/Prebiotic Supplementation}

Probiotics are living and non-pathogenic microorganisms that are taken with or separately from food, regulate the intestinal flora and stimulate the immune system and positively affect host health (31). Fermented yoghurt, cheese, pickle, sausage, bread, beer, wine, chicken and kefir are the nutritional sources of probiotics (32). Prebiotics are called undigested carbohydrates (33). Prebiotics are necessary for feeding of microorganisms which are beneficial for our body (31).

Probiotics can modulate the gastrointestinal luminal immunity by changing the cellular environment from the proinflammatory to the anti-inflammatory state (12). They can also convert undigested carbohydrates into short-chain fatty acids. It is claimed that probiotics can lead to symptomatic improvements in IBS patients and that each strain of bacteria can affect selected symptoms (3).

Numerous studies investigating the effectiveness of probiotics in patients with IBS have been conducted. Although most of these studies are double-blind randomized controlled, the different diagnostic criteria of IBS, the difference in the probiotic type used, its dose, delivery intervals, duration of study, and patient groups prevent the studies from being compared $(34,35)$.

Didari et al. (36) investigated the efficiency of probiotics in IBS patients in 2015 and demonstrated that probiotics reduced the pain and symptom severity scores and had beneficial effects compared to placebo. In the study of Šmid et al. (37) in 2016, IBS patients were given probiotic yoghurt and dietary fiber for eight weeks, and as a result of the study, it was reported that there was averagely $18 \%$ improvement in the patients' quality of life scores (decrease in bloating severity, bowel movements, etc.).

Nowadays, it is recommended to use prebiotics with other treatment options because of the lack of effective treatment in IBS patients and the probiotics' being safe. IBS patients using probiotics should be advised to choose one product at the same time and monitor its effects. As a result of a metaanalysis, probiotics should be used at the dosage of the manufacturer's recommendations, and it has been proposed to be used for at least four weeks so that the efficacy can occur (38).

There are few randomized controlled trials that examine the effects of prebiotic use in patients with IBS. In the study of Olesen and Gudmand-Hoyer (39), IBS patients were given
$22 \mathrm{~g} /$ day fructooligosaccharide for 12 weeks, and it was found that these high doses increased the complaints of patients in 4-6 weeks. Silk et al. (40) gave a dose of 3.5 and $7 \mathrm{~g} /$ day galactooligosaccharide to 60 IBS patients for four weeks, and at the end of the treatment, they found significant improvement in both general IBS symptoms and symptoms of bloating in patients receiving low-dose prebiotic therapy.

\section{Conclusion}

In recent years, healthy nutrition and lifestyle changes have gained great importance in the treatment of IBS. There are studies suggesting that especially soluble fiber reduces symptoms and improves quality of life in patients with IBS. Nowadays, there is increasing evidence to support the use of a low FODMAP diet in most patients with IBS. Another approach which is important in IBS management is the use of probiotics. However, it is not yet clear which strain and dose are beneficial. Therefore, in order to verify and assess the approach of increasing fiber in diet, having low FODMAP diet and using probiotic in the long term, further well-designed and randomized controlled trials are needed.

\section{Ethics}

Peer-review: Externally peer-reviewed.

\section{Authorship Contributions}

Concept: H.E., E.N.Ü., G.A., Design: H.E., E.N.Ü., G.A., Data Collection or Processing: E.N.Ü., Analysis or Interpretation: H.E., Literature Search: E.N.Ü., Writing: H.E., E.N.Ü.

Conflict of Interest: No conflict of interest was declared by the authors.

Financial Disclosure: The authors declared that this study received no financial support.

\section{References}

1. Werlang ME, Palmer WC, Lacy BE. Irritable Bowel Syndrome and Dietary Interventions. Gastroenterol Hepatol. 2019;15:16-26.

2. Nanayakkara WS, Skidmore PM, O'Brien L, Wilkinson TJ, Gearry RB. Efficacy of the low FODMAP diet for treating irritable bowel syndrome: the evidence to date. Clin Exp Gastroenterol. 2016;9:131-142.

3. Brenner DM, Moeller MJ, Chey WD, Schoenfeld PS. The utility of probiotics in the treatment of irritable bowel syndrome: a systematic review. Am J Gastroenterol. 2009;104:1033-1049.

4. Dimidi E, Rossi M, Whelan K. Irritable bowel syndrome and diet: where are we in 2018? Curr Opin Clin Nutr Metab Care. 2017;20:456-463.

5. Cozma-Petruţ A, Loghin F, Miere D, Dumitraşcu DL. Diet in irritable bowel syndrome: What to recommend, not what to forbid to patients! World J Gastroenterol. 2017;23:3771-3783. 
6. McKenzie YA, Bowyer RK, Leach H, et al. British Dietetic Association systematic review and evidence-based practice guidelines for the dietary management of irritable bowel syndrome in adults (2016 Update) J Hum Nutr Diet. 2016;29:549-575.

7. Camilleri M. Management Options for Irritable Bowel Syndrome. Mayo Clin Proc. 2018;93:1858-1872.

8. El-Salhy M, Ystad SO, Mazzawi T, Gundersen D. Dietary fiber in irritable bowel syndrome (Review). Int J Mol Med. 2017;40:607-613.

9. Portincasaa P, Lembob A, Baria O, et al. The Role of Dietary Approach in Irritable Bowel Syndrome. Curr Med Chem. 2019;26:3512-3520.

10. Chey WD, Maneerattaporn M, Saad R. Pharmacologic and complementary and alternative medicine therapies for irritable bowel syndrome. Gut Liver. 2011;5:253-266.

11. Lee BJ, Bak YT. Irritable bowel syndrome, gut microbiota and probiotics. J Neurogastroenterol Motil. 2011;17:252266.

12. Dale HF, Rasmussen SH, Asiller ÖÖ, Lied GA. Probiotics in Irritable Bowel Syndrome: An Up-to-Date Systematic Review'. Nutrients. 2019;11:2048.

13. Staudacher HM, Lomer MC, Anderson JL, et al. Fermentable Carbohydrate Restriction Reduces Luminal Bifidobacteria and Gastrointestinal Symptoms in Patients with Irritable Bowel Syndrome. J Nutr. 2012;142:1510-1518.

14. Staudacher HM, Whelan K, Irving PM, Lomer MCE. Comparison of symptom response following advice for a diet low in fermentable carbohydrates (FODMAPs) versus standard dietary advice in patients with irritable bowel syndrome. J Hum Nutr Diet. 2011;24:487-495.

15. Enck P, Aziz Q, Barbara G, et al. Irritable bowel syndrome. Nat Rev Dis Primers. 2016;2:16014.

16. Spiller R, Aziz Q, Creed F, et al. Guidelines on the irritable bowel syndrome: mechanisms and practical management. Gut. 2007;56:1770-1798.

17. Lacy BE. The Science, Evidence, and Practice of Dietary Interventions in Irritable Bowel Syndrome. Clinical Gastroenterology and Hepatology. 2015;15:16-26.

18. Guo YB, Zhuang KM, Kuang L, Zhan Q, Wang XF, Liu SD. Association between Diet and Lifestyle Habits and Irritable Bowel Syndrom A Case-Control Study. Gut Liver. 2015;9:649-656.

19. Şahan Y, Dülger D. Diyet Lifin Özellikleri ve Sağlık Üzerindeki Etkileri. Uludağ Üniversitesi Ziraat Fakültesi Dergisi. 2011;25:147-157.

20. Deehan EC, Walter J. The fiber gap and the disappearing gut microbiome: implications for human nutrition. Trends Endocrinol Metab. 2016;27:239-242.

21. McRorie JW. Evidence-based approach to fiber supplements and clinically meaningful health benefit Part 2: What to look for and how to recommend an effective fiber therapy. Nutr Today. 2015;50:90-97.
22. Moayyedi P, Quigley EM, Lacy BE, et al. The effect of fiber supplementation on irritable bowel syndrome: A systematic review and meta-analysis. Am J Gastroenterol. 2014;109:1367-1374.

23. Ishiguro, E, Haskey, N, Campbell, K. Gut microbiota interactive effect on nutrition and health'. 1st Edition. Academic Press. 2018.

24. Cuomo R, Andreozzi P, Zito FP, Passananti V, De Carlo G, Sarnelli G. Irritable bowel syndrome and food interaction. World J Gastroenterol. 2014;20:8837-8845.

25. Halmos EP, Christophersen CT, Bird AR, Shepherd SJ, Gibson PR, Muir JG. Diets that differ in their FODMAP content alter the colonic luminal microenvironment. Gut. 2015;64:93-100.

26. Gibson PR, Shepherd SJ. Evidence-based dietary management of functional gastrointestinal symptoms: The FODMAP approach. J Gastroenterol Hepatol. 2010;25:252258.

27. Fasano A, Sapone A, Zevallos V, Schuppan D. Nonceliac Gluten Sensitivity. Gastroenterology. 2015;148:1195-1204.

28. Halmos EP, Power VA, Shepherd SJ, Gibson PR, Muir JG. A diet low in FODMAPs reduces symptoms of irritable bowel syndrome. Gastroenterology. 2014;146:67-75.

29. Mclntosh K, Reed DE, Schneider T, et al. FODMAPs alter symptoms and the metabolome of patients with IBS: a randomised controlled trial. Gut. 2017;66:1241-1251.

30. Tuck C, Barrett J. Re-challenging FODMAPs: the low FODMAP diet phase two. J Gastroentrol Hepatol. 2017;32(Suppl 1):11-15.

31. Coşkun T. Pro- Pre- Sinbiyotikler. Çocuk Sağlığı ve Hastalıkları Dergisi. 2006;49:128-148.

32. Yağcı R. Prebiyotikler ve Probiyotikler. Çocuk Sağlığı Ve Hastalıkları Dergisi. 2002;45:337-344.

33. Özden A. Gastrointestinal Sistem ve Probiyotik-Prebiyotik Synbiyotik. Güncel Gastroenteroloji. 2005;9:124-133.

34. Rogers NJ, Mousa SA. The Shortcomings of clinical trials assessing the Efficiacy of probiotics in Irritable bowel syndrome. J Altern Complement Med. 2012;18:112-119.

35. Whelan K. Probiotics and prebiotics in the management of Irritable bowel syndrome: a review of recent clinical trials and systematic reviews. Curr Opin Clin Nutr Metab Care. 2011;14:581-587.

36. Didari T, Mozaffari S, Nikfar S, Abdollahi M. Effectiveness of probiotics in irritable bowel syndrome: Updated systematic review with meta-analysis. World J Gastroenterol. 2015;21:3072-3084.

37. Šmid A, Strniša L, Bajc K, Vujić-Podlipec D, Matijašić BB, Rogelj I. (2016). Randomized clinical trial: The effect of fermented milk with the probiotic cultures Lactobacillus acidophilus La- $5 \AA$ and Bifidobacterium BB-12® and Beneo dietary fibres on health-related quality of life and the symptoms of irritable bowel syndrome in adults. Journal of Functional Foods.2016;24:549-557. 
38. Pasqui F, Poli C, Magrino C, Festi D. Dietary Management in IBS Patients. Irritable Bowel Syndrome - Novel Concepts For Research And Treatment. 2016.

39. Olesen M, Gudmand-Hoyer E. Efficacy, safety and tolerability of fructooligosaccharides of in the treatment of Irritable bowel syndrome. Am J Clin Nutr. 2000;72:15701575.
40. Silk DB, Davis A, Vulevic J, Tzortzis G, Gibson GR. Clinical Trial: the effects of a trans-galactoolisaccharide prebiotic on faecal microbiota and symtoms in Irritable bowel syndrome. Aliment Pharmacol Ther. 2009;29:508-518. 